\title{
Preclinical analysis of MTOR complex 1/2 inhibition in diffuse intrinsic pontine glioma
}

\author{
PATRICK C. FLANNERY ${ }^{1 *}$, JOHN A. DESISTO ${ }^{1 *}$, VLADIMIR AMANI $^{1}$, SUJATHA VENKATARAMAN $^{1}$, \\ RAKEB T. LEMMA ${ }^{1}$, ERIC W. PRINCE ${ }^{1}$, ANDREW DONSON ${ }^{1}$, ERIN E. MOROZE ${ }^{1}$, LINDSEY HOFFMAN $^{1,2}$, \\ JEAN M. MULCAHY LEVY ${ }^{1,2}$, NICHOLAS FOREMAN ${ }^{1,2}$, RAJEEV VIBHAKAR $^{1,2}$ and ADAM L. GREEN ${ }^{1,2}$ \\ ${ }^{1}$ Morgan Adams Foundation Pediatric Brain Tumor Research Program, University of Colorado School of Medicine; \\ ${ }^{2}$ Center for Cancer and Blood Disorders, Children's Hospital Colorado, Aurora, CO 80045, USA
}

Received April 11, 2017; Accepted November 21, 2017

DOI: $10.3892 / o r .2017 .6122$

\begin{abstract}
Diffuse intrinsic pontine glioma (DIPG) is an incurable childhood brain tumor. The mechanistic target of rapamycin (MTOR), a key oncogene, functions as two distinct signaling complexes, MTORC1 and MTORC2. We set out to determine the preclinical efficacy and mechanism of action of MTOR inhibitors in DIPG. We evaluated the MTORC1 inhibitor everolimus and the MTORC1/2 inhibitor AZD2014 in three patient-derived DIPG cell lines using cell culture models. We created dose-response curves for both compounds. We measured phenotypic effects on cell selfrenewal, apoptosis, cell cycle, differentiation, senescence, and autophagy. We assessed the effects of each compound on the AKT pathway. Finally, we measured the efficacy of AZD2014 in combination with radiation therapy (RT) and a panel of FDA-approved chemotherapy drugs. While everolimus showed minimal antitumor efficacy, AZD2014 revealed $\mathrm{IC}_{50}$ levels of $410-552 \mathrm{nM}$ and $\mathrm{IC}_{90}$ levels of $1.30-8.86 \mu \mathrm{M}$ in the three cell lines. AZD2014 demonstrated increased inhibition of cell self-renewal compared to everolimus. AZD2014 decreased expression of phospho-AKT, while no such effect
\end{abstract}

Correspondence to: Dr Adam L. Green, Morgan Adams Foundation Pediatric Brain Tumor Research Program, University of Colorado School of Medicine, Anschutz Medical Campus, 12800 E. 19th Avenue, Mail Stop 8302, Aurora, CO 80045, USA

E-mail: adam.green@ucdenver.edu

*Contributed equally

Abbreviations: AKT, V-Akt murine thymoma viral oncogene; BBB, blood-brain barrier; DIPG, diffuse intrinsic pontine glioma; GSEA, gene set enrichment analysis; MTOR, mechanistic target of rapamycin; MTORC1, MTOR complex 1; MTORC2, MTOR complex 2; RICTOR, RPTOR independent companion of MTORC2; RPTOR, regulatory associated protein of MTORC1; RT, radiation therapy; SEM, standard error of the mean

Key words: diffuse intrinsic pontine glioma, MTOR, MTORC1, MTORC2, neurosphere culture, cell self-renewal, AKT, radiation therapy, ponatinib, combination therapy was noted with everolimus. Direct AKT inhibition showed similar efficacy to AZD2014, and induction of constitutive AKT activity rescued DIPG cells from the effects of AZD2014. AZD2014 exhibited synergistic relationships with both RT and various chemotherapy agents across classes, including the multikinase inhibitor ponatinib. MTORC1/2 inhibition shows antitumor activity in cell culture models of DIPG due to the effect of MTORC2 inhibition on AKT. This strategy should be further assessed for potential incorporation into combinatorial approaches to the treatment of DIPG.

\section{Introduction}

Diffuse intrinsic pontine glioma (DIPG) is an incurable brain tumor that mostly affects young children (1). Treatment with radiation therapy (RT) is usually transiently effective, but median progression-free survival is only 7 months, and median overall survival is 11 months (2). Hundreds of clinical trials studying systemically delivered cytotoxic and targeted chemotherapy agents have been conducted without showing any clinical benefit, potentially due to inadequate tumor penetration (3). Investigators are therefore studying novel drug delivery methods for these patients $(4,5)$, and some of these have reached clinical trials $(6,7)$. As these studies advance, better targeted therapeutic options will be needed, especially as part of combination treatments.

The mechanistic target of rapamycin (MTOR) is a serinethreonine kinase that has crucial roles in many cellular pathways dysregulated in cancer, including metabolism, growth, survival, and response to stress. It functions as part of two distinct signaling complexes, MTOR complex 1 (MTORC1), which also contains the regulatory associated protein of MTORC1 (RPTOR), and MTOR complex 2 (MTORC2), containing the RPTOR independent companion of MTORC2 (RICTOR). MTORC2 activates the V-Akt murine thymoma viral oncogene (AKT) through phosphorylation at S473, which mediates many of the cellular effects of MTORC2 (8-10). Preclinical and clinical data suggest a role for MTOR in gliomagenesis (11). First generation MTOR inhibitors (rapamycin analogues or rapalogs), such as everolimus, inhibit only MTORC1, and their clinical use in high-grade glioma has been disappointing (12). Contrastingly, Kahn et al 
reported preclinical findings showing that the MTORC1/2 inhibitor AZD2014 enhanced the efficacy of RT in adult glioblastoma stem cells in vitro and in vivo (13), generating hope that combined MTOR inhibition may hold more clinical promise. DIPG has significant biological differences from adult glioblastoma, however $(14,15)$, and prior to undertaking our project, MTOR inhibition of any kind in DIPG had not been addressed in the literature.

In this study, we examined the effects of MTOR inhibition preclinically in DIPG. We hypothesized that the addition of MTORC2 inhibition would increase the antitumor efficacy over the targeting of MTORC1 alone. Our overall goal was to determine how MTOR inhibition could contribute to future clinical treatment approaches to this devastating tumor.

\section{Materials and methods}

Aim and design. The aim of this study was to determine the efficacy of MTORC1/2 compared to MTORC1 inhibitors in DIPG. We set out to determine the phenotypic and molecular basis for the difference in efficacy noted between inhibitors. We then studied how MTORC1/2 inhibition could be used in combination with other existing treatments. All assays took place using three patient-derived short-term culture cell lines in cell culture models.

Gene expression in tumor bank. We performed gene expression profiling on patient-derived DIPG $(\mathrm{n}=16)$ and normal pons $(n=2)$ samples. All tumor samples were collected from consented pediatric patients undergoing tumor biopsy using an IRB-approved study protocol (COMIRB 95-500). Samples were snap frozen and stored in liquid nitrogen. RNA was extracted from each sample using an RNeasy or DNA/RNA AllPrep kit (Qiagen, Valencia, CA, USA) according to the manufacturer's instructions. Four hundred nanograms of RNA was processed using the Ambion MessageAmp ${ }^{\mathrm{TM}}$ Premier RNA Amplification kit (Applied Biosystems, Foster City, CA, USA), according to the manufacturer's instructions. RNA quality was verified using the Nano Assay Protocol for the 2100 Bioanalyzer (Agilent, Santa Clara, CA, USA) at two time-points: i) after initial extraction of the RNA from the tumor sample, and ii) after preparation of the RNA for chip hybridization. The prepared RNA was hybridized to HG-U133 Plus 2 GeneChips (Affymetrix, Santa Clara, CA, USA), according to the manufacturer's instructions to measure gene expression. Mean and standard error of fold changes for each sample set were calculated for each measure indicated. We also conducted hallmark gene set enrichment analysis (GSEA) on the sample sets.

Gene expression from public data. We performed gene expression analysis using the R2 genomics analysis and visualization platform (http://r2.amc.nl) using the default settings. We compared expression of MTOR, RPTOR, and RICTOR in a set of normal brain samples ( $n=172$, Berchtold set) versus DIPG samples ( $\mathrm{n}=37$, Paugh set) using one way analysis of variance.

Tumor lines and culture conditions. Three primary human pediatric (DIPG 4 and DIPG 6, derived from previously
Table I. Cell line characteristics.

\begin{tabular}{lllc}
\hline Cell line & H3 mutation & \multicolumn{1}{c}{$\begin{array}{c}\text { Other genetic } \\
\text { features }\end{array}$} & $\begin{array}{c}\text { When } \\
\text { collected }\end{array}$ \\
\hline DIPG 4 & H3. 1K27M & $\begin{array}{l}\text { TP53 WT, } \\
\text { ACVR1 G328V } \\
\text { TP53 mutant }\end{array}$ & Autopsy \\
DIPG 6 & H3.3K27M & $\begin{array}{l}\text { Autopsy } \\
\text { Exogenous hTERT }\end{array}$ & Biopsy \\
\hline
\end{tabular}

irradiated DIPGs at autopsy, provided by Dr Michelle Monje, Stanford University, and SF7761, derived from a biopsy sample, provided by Dr Nalin Gupta, University of California, San Francisco) were grown in neurosphere (suspension) culture conditions in ultra-low attachment flasks (Corning, Corning, NY, USA, DIPG 4 and DIPG 6) or in tissue culturetreated flasks (SF7761; Falcon/Corning) (16). Characteristics of the cell lines are listed in Table I (17). For certain experiments, DIPG 4 cells were also grown adherently in tissue culture-treated plates. The identity of all lines was validated by molecular profiling and compared to known results prior to and during this project. The cell lines were maintained in Neurobasal-A medium mixed 1:1 with Dulbecco's modified Eagle's medium/F-12 supplemented 1:100 by volume with HEPES [4-(2-hydroxyethyl)-1-piperazine ethanesulfonic acid] $1 \mathrm{M}$, sodium pyruvate $100 \mathrm{mM}$, MEM non-essential amino acids $10 \mathrm{mM}$, GlutaMAX-I, and antibiotic-antimycotic (all Gibco/Life Technologies, Waltham, MA, USA); B27-A supplement 50X (1:50; Invitrogen), heparin ( $2 \mu \mathrm{g} / \mathrm{ml}$; Stemcell Technologies, Inc., Vancouver, BC, Canada) and human EGF, $\mathrm{FGFb}$, and platelet derived growth factor-AB (all $20 \mathrm{ng} / \mathrm{ml}$; Shenandoah Biotech, Warwick, PA, USA). For SF7761 cells, N2 supplement was also added (1:100; Life Technologies). For all experiments, except as noted, cells were plated at a concentration of 200,000 cells $/ \mathrm{ml}$ of media in ultra-low attachment plates (Corning). Prior to all end-point measurements, neurospheres were dispersed by trituration using a micropipette.

Dose-response curves. Cell proliferation was determined by the MTS [3-(4, 5-dimethylthiazol-2-yl)-5-(3-carboxymethoxyphenyl)-2-(4-sulfophenyl)-2H-tetrazolium] assay using CellTiter 96 AQueous One Solution (Promega, Madison, WI, USA). Cells were seeded at 5,000 cells per well into a 96-well plate (Costar) and allowed to attach overnight (DIPG 4) or in a 96-well plate (Corning) in neurosphere culture (DIPG 6 and SF7761), in a total volume of $100 \mu 1$ of media. Twenty-four hours later, the cells were treated with a range of doses of everolimus and AZD2014 (Selleck, Houston, TX, USA) in triplicate. At the end of the drug treatment period $(72 \mathrm{~h}$ for DIPG 4 and DIPG 6, $120 \mathrm{~h}$ for SF7761), $20 \mu \mathrm{l}$ of MTS reagent was added to each well to make a final volume of $120 \mu \mathrm{l}$ and allowed to develop. Absorbance values for plate wells were acquired using a BioTek Synergy 2 plate reader at a wavelength of $490 \mathrm{~nm}$ after $3 \mathrm{~h}$ of incubation, and background absorbance was subtracted. $\mathrm{IC}_{50}$ values were determined experimentally through Prism, and $\mathrm{IC}_{90}$ values were calculated using the $\mathrm{IC}_{50}$ and hill slope value using the Graphpad EC Anything online calculator. 
Neurosphere dilution assay. DIPG 4 and SF7761 cells were plated in 96-well format in suspension, with 10 cells per well and 20 wells per condition. Conditions for each cell line included DMSO control, the calculated $\mathrm{IC}_{75}$ for AZD2014, or the same concentration of everolimus. The presence and size (area using two perpendicular axes) of a neurosphere in each well was measured after three weeks.

Apoptosis measurement. SF7761 cells in neurosphere culture were treated in a 96-well plate format with the indicated range of AZD2014 and everolimus concentrations compared to the control, in triplicate. Apoptosis was measured according to the manufacturer's instructions using the Caspase Glo 3/7 assay (Promega) at $48 \mathrm{~h}$ of treatment after $2 \mathrm{~h}$ of incubation with the Caspase-Glo reagent at room temperature.

Cell cycle analysis. SF7761 cells in neurosphere culture were treated in a 6-well format with the indicated range of AZD2014 and everolimus concentrations compared to the control for $24 \mathrm{~h}$. Cells were then removed from compound solutions and fixed with $1 \mathrm{ml}$ ice cold $70 \%$ ethanol dropwise with gentle vortexing. Cells were incubated overnight at $4^{\circ} \mathrm{C}$. After $24 \mathrm{~h}$, the cells were removed from $70 \%$ ethanol and placed into $200 \mu 1$ of propridium iodide (PI) and incubated for $30 \mathrm{~min}$. The stained cells then underwent cell cycle analysis by flow cytometry with a Guava EasyCyte (Millipore, Billerica, MA, USA). Data analysis was carried out using FlowJo (Ashland, OR, USA).

Differentiation analysis. DIPG 4 cells were plated into 8-well chamber slides treated with Poly-D-lysine to improve cell adhesion (354632; Corning BioCoat) in $0.5 \mathrm{ml}$ media per well. The cells were allowed to gain adhesion overnight and were then treated for $72 \mathrm{~h}$ with the indicated range of everolimus and AZD2014 concentrations compared to the control. Following treatment, the cells were fixed, permeabilized, and co-stained for TUBB3 (MAB1195; R\&D Systems) and GFAP (ag7260; Abcam), followed by conjugation respectively to Alexa Fluor 555 and Alexa Fluor 488 fluorescent secondary antibodies. Final staining with DAPI was performed to delineate nuclei. Confocal images were acquired at a magnification of 400x using a Zeiss Axio Observer Z1 microscope with Yokogawa CSU-X1 camera and lasers of 405 (DAPI), 488 and $561 \mathrm{~nm}$ wavelength for fluorophore excitation. Mean values of fluorescence intensity were computed on a cell by cell basis using Image J and then averaged.

Senescence measurement by p21 immunofluorescence. Adherently growing DIPG 4 cells were plated at a density of 20,000 cells per well in BioCoat 8-well chamber slides coated with Poly-D-lysine and laminin (Corning) and allowed $\sim 24 \mathrm{~h}$ in which to develop adhesion before subjecting them to experimental conditions. SF7761 cells were plated at a density of 1,000,000 cells per well of a 6-well plate (Corning). Cells were treated for two days with $50 \mathrm{nM}, 500 \mathrm{nM}$ or $5 \mu \mathrm{M}$ of AZD2014 or everolimus, along with DMSO control. After two days of incubation, the cells were placed into normal growth media for five days. Cells were fixed for $20 \mathrm{~min}$ in $37 \%$ formaldehyde diluted in 10X PBS (Sigma, St. Louis, MO, USA), permeabilized in $0.1 \%$ Triton $\mathrm{X}$ (Sigma) in PBS for
$10 \mathrm{~min}$, and blocked for $45 \mathrm{~min}$ in $4 \%$ bovine serum albumin (BSA) in PBS supplemented with $0.05 \%$ Triton X. Cells were then incubated in primary antibody to p21 (2947S; 1:200; Cell Signaling Technology, Inc.), with 4\% BSA (in PBS) and $0.05 \%$ Triton $\mathrm{X}$, for $1 \mathrm{~h}$ at room temperature or overnight at $4^{\circ} \mathrm{C}$. SF7761 cells to be stained were fixed and permeabilized in Foxp3/Transcription Factor Fixation/Permeabilization Concentrate and Diluent (Affymetrix EBioscience, Santa Clara, CA, USA) according to the manufacturer's instructions. Cells were incubated in primary antibody to p21, along with Permeabilization Buffer (Affymetrix EBioscience). After rinsing to remove the primary antibody, adherent and suspension cells were incubated in secondary antibody (1:500; Alexa Fluor 488; Life Technologies) for $1 \mathrm{~h}$ at room temperature. Suspension cells were affixed to slides using a Cytospin 4 Centrifuge (Thermo Fisher Scientific) at 2,000 rpm for $10 \mathrm{~min}$. Slides were fixed using ProLong antifade reagent with DAPI (Life Technologies). Confocal imaging was performed at a magnification of x400 using 405 (DAPI) and $488 \mathrm{~nm}$ (Alexa Fluor 488) lasers on a 3I Marianas imaging system (Intelligent Imaging Innovations). Images were obtained using an Evolve 16-bit EMCCD camera (Photometrics, Tucson, AZ, USA).

Senescence measurement by $\beta$-galactosidase staining. Adherently growing DIPG 4 cells were plated at a density of 100,000 cells per well in a 6-well plate and allowed $\sim 24 \mathrm{~h}$ in which to develop adhesion before subjecting them to experimental conditions. Cells were treated for two days with $50 \mathrm{nM}$, $500 \mathrm{nM}$ or $5 \mu \mathrm{M}$ of AZD2014 or everolimus, along with DMSO control. After two days of incubation, the cells were placed into normal growth media for five days. Staining for $\beta$-galactosidase was performed using a Senescence $\beta$-galactosidase Staining kit (Cell Signaling Technology, Inc.) according to the manufacturer's instructions. Cells were washed with $2 \mathrm{ml}$ of PBS, followed by a 15 -min incubation using $1 \mathrm{X}$ fixative. Cells were again washed twice with PBS and then incubated with $1 \mathrm{ml}$ of $\beta$-galactosidase staining solution. The cells were incubated overnight in a dry $37^{\circ} \mathrm{C}$ incubator without $\mathrm{CO}_{2}$. Senescencent cells were imaged via brightfield microscopy (Nikon DS-L2).

Autophagy measurement. DIPG 4 and SF7761 cells were plated in neurosphere culture in 6-well plates and exposed to one of the following conditions for $4 \mathrm{~h}$ : $0.2 \%$ DMSO, $10 \mu \mathrm{M}$ chloroquine (MP Biomedicals), $1 \mu \mathrm{M}$ AZD2014, $1 \mu \mathrm{M}$ everolimus, $1 \mu \mathrm{M}$ AZD2014 combined with $10 \mu \mathrm{M}$ chloroquine, or $1 \mu \mathrm{M}$ everolimus combined with $10 \mu \mathrm{M}$ chloroquine. Immediately following treatment, the cells were lysed, and western blotting was conducted as described below.

AKT/phospho-AKT measurement. DIPG 4 and SF7761 cells in neurosphere culture were treated in a 6-well format with the indicated range of AZD2014 and everolimus versus the control for $2 \mathrm{~h}$. Western blotting was then conducted as below using antibodies specific to phosphorylated and unphosphorylated AKT.

Western blotting. Cells were lysed in $200 \mu \mathrm{l}$ radioimmunoprecipitation assay buffer with 100X protease and phosphatase inhibitor (Pierce; Thermo Fisher Scientific) by gentle vortexing for $5 \mathrm{sec}$. Cells were incubated on ice for $5 \mathrm{~min}$ before 
centrifugation at $4^{\circ} \mathrm{C}$ at $14,000 \mathrm{x}$ g for $10 \mathrm{~min}$. Protein concentration was measured using the Pierce BCA assay. Pre-cast 4-20\% Mini-PROTEAN TGX gels (Bio-Rad, Hercules, CA, USA) were loaded with $\sim 30 \mu \mathrm{g}$ of protein per lane and then run for $90 \mathrm{~min}$ at $125 \mathrm{~V}$. Transfer was then performed to a polyvinylidine fluoride membrane via a Bio-Rad transfer set at $4^{\circ} \mathrm{C}$ at $50 \mathrm{~V}$ for $90 \mathrm{~min}$. Blocking was carried out in $5 \%$ non-fat dry milk, as was all staining. Primary staining was performed as indicated for $\alpha$-tubulin (\#2125 rabbit anti-human $\mathrm{mAb}$ at 1:1,000 overnight at $4^{\circ} \mathrm{C}$; Cell Signaling Technology, Inc.); pAKT (\#4060 rabbit anti-human $\mathrm{mAb}$ at 1:1,000 overnight at $4^{\circ} \mathrm{C}$; Cell Signaling Technology, Inc.); and total AKT (\#9272 rabbit anti-human pAb at 1:1,000 overnight at $4^{\circ} \mathrm{C}$; Cell Signaling Technology, Inc.). Secondary staining was carried out with anti-rabbit HRP at 1:3,000 for $1 \mathrm{~h}$ at room temperature. Blots were developed using Western Lightning Plus-ECL chemiluminescent (PerkinElmer, Waltham, MA, USA).

AKT small molecule inhibition. DIPG 4 and SF7761 cells in neurosphere culture were treated in a 96-well format in triplicate at the indicated concentration range of MK-2206 (Selleck Chemicals, Houston, TX, USA) compared to the control for $120 \mathrm{~h}$. Cell viability was then determined by MTS assay as above.

Constitutive AKT activation. Introduction of a mutation from serine to aspartate at amino acid 473 of AKT (S473D) mimics serine phosphorylation at this position and results in constitutive activation of the protein (18). DIPG 4 cells were plated at a density of 200,000 cells per well in $2 \mathrm{ml}$ of medium in a 6-well plate and allowed to adhere overnight. AKT cDNA containing the S473D mutation (gift of Dr N. Rosen) was transfected into the cells using jetPRIME ${ }^{\circledR}$ transfection reagent $(2 \mu \mathrm{g}$ cDNA per well mixed into $200 \mu \mathrm{l}$ jetPRIME buffer, followed by $4 \mu \mathrm{l}$ of jetPRIME reagent; 5-min incubation; Thermo Fisher). The medium was changed at $18 \mathrm{~h}$ following transfection. At $48 \mathrm{~h}$ following transfection, western blotting was conducted as above. Transfected and wild-type cells were then plated in a 96-well format, allowed to adhere, and then treated for $120 \mathrm{~h}$ as follows with the indicated range of AZD2014 concentrations compared to the control, in sextuplicate. Cell viability was then measured by MTS assay as above.

Radiation combination. SF7761 cells in neurosphere culture were plated in a 96-well format in triplicate. The next day, they were exposed to irradiation at the indicated dose range using a cesium irradiator. They were then treated starting the next day with the indicated dose range of AZD2014 compared to control for $120 \mathrm{~h}$. Cell viability was then measured by MTS assay as above. The combination index at each dose level combination was determined by the Chou Talalay method (19).

Immunofluorescence staining. Immunofluorescence staining for $\gamma \mathrm{H} 2 \mathrm{AX}$ was performed on cells exposed to AZD2014 followed by radiation to identify double-stranded DNA (dsDNA) damage patterns. SF7761 cells were plated at a density of 20,000 cells per well in BioCoat Poly-D-lysine/laminincoated chamber slides (\#354688; Corning) and allowed $24 \mathrm{~h}$ in which to develop adhesion before subjecting them to experimental conditions. Cells were treated with vehicle or AZD2014 at 50 and $500 \mathrm{nM}$ dose levels for 4 or $48 \mathrm{~h}$ and then irradiated with 4 Gy from a $\mathrm{Cs}^{137}$ source in a single dose. At $4 \mathrm{~h}$ postradiation, the cells were fixed for $20 \mathrm{~min}$ in $37 \%$ formaldehyde diluted in 10X PBS (Sigma), permeabilized in $0.1 \%$ Triton X in PBS for $10 \mathrm{~min}$, and blocked for $45 \mathrm{~min}$ in 4\% BSA in PBS supplemented with $0.05 \%$ Triton $\mathrm{X}$. Cells were incubated in primary $\gamma \mathrm{H} 2 \mathrm{AX}$ antibody (\#2577; Cell Signaling Technology, Inc.) diluted 1:600 with 4\% BSA, dissolved in PBS and 0.05\% Triton $\mathrm{X}$, for $1 \mathrm{~h}$ at room temperature. After rinsing, cells were incubated in secondary antibody (1:500; Alexa Fluor 488; Life Technologies) for $1 \mathrm{~h}$ at room temperature. Slides were fixed using ProLong antifade reagent with DAPI (Life Technologies). Confocal imaging was performed at a magnification of $x 400$ using 405 (DAPI) and $488 \mathrm{~nm}$ (Alexa Fluor 488) lasers on a 3I Marianas imaging system (Intelligent Imaging Innovations). Images were obtained using an Evolve 16-bit EMCCD camera (Photometrics).

Chemotherapy combination panel. SF7761 cells in neurosphere culture were plated in a 96-well format. One set of cells was treated with the $\mathrm{IC}_{50}$ concentration of AZD2014, while another set of cells was treated with DMSO. All drugs from the Approved Oncology Drugs Set VII (National Cancer Institute, Bethesda, MD, USA) were then added to one well of each set of cells at a concentration of $1 \mu \mathrm{M}$. In addition, 6 wells of cells were treated with the $\mathrm{IC}_{50}$ concentration of AZD2014 alone, and 6 with DMSO alone. Cells were exposed to these compounds for $120 \mathrm{~h}$, and then cell viability was determined by MTS assay as above. The relative cell viability for each combination was then compared to DMSO alone. The therapeutic relationship for each combination was then calculated by dividing this value by the product of the relative cell viability for each drug and the relative cell viability for AZD2014 alone.

Ponatinib combination. DIPG 4, DIPG 6, and SF7761 cells were treated in a 96-well format in triplicate with the indicated ranges of AZD2014, ponatinib, and the two compounds together, compared to the control, for $120 \mathrm{~h}$. Cell viability was then determined by MTS assay as above. The combination index at each dose level combination was determined by the Chou Talalay method (19).

Data analysis. All data analysis and chart creation was performed using GraphPad Prism. All charts show mean \pm standard error of the mean (SEM) unless otherwise noted. Comparison of the percentage of neurospheres in the neurosphere dilution assay was carried out via Chi-square comparison of proportions test. Comparison of mean neurosphere area was performed by the unpaired t-test. Statistical significance is represented as follows: $p>0.05$ is indicated by ns (not significant); $\mathrm{p}=0.05-0.01$ is indicated by $* ; \mathrm{p}=0.01$ 0.001 is indicated by **; $\mathrm{p}=0.001-0.0001$ is indicated by $* * *$; $\mathrm{p}<0.0001$ is indicated by $* * * *$.

\section{Results}

Variable MTOR expression in human DIPG samples versus normal brain. We first performed measurements of gene expression of MTOR and the MTOR components RPTOR (MTORC1) and RICTOR (MTORC2) in a panel of human 


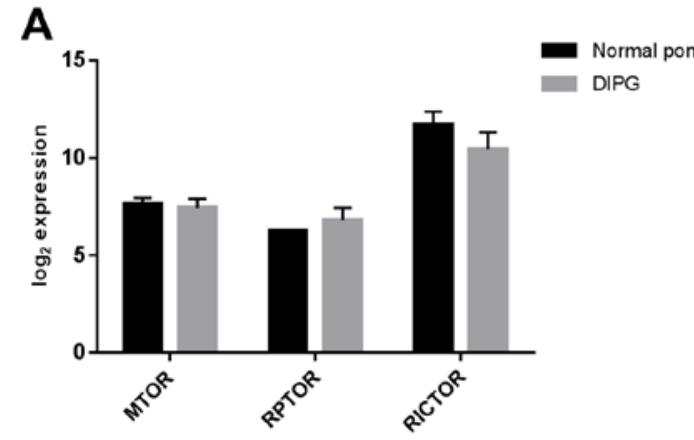

B Enrichment plot: HALLMARK_MTORC1_SIGNALING
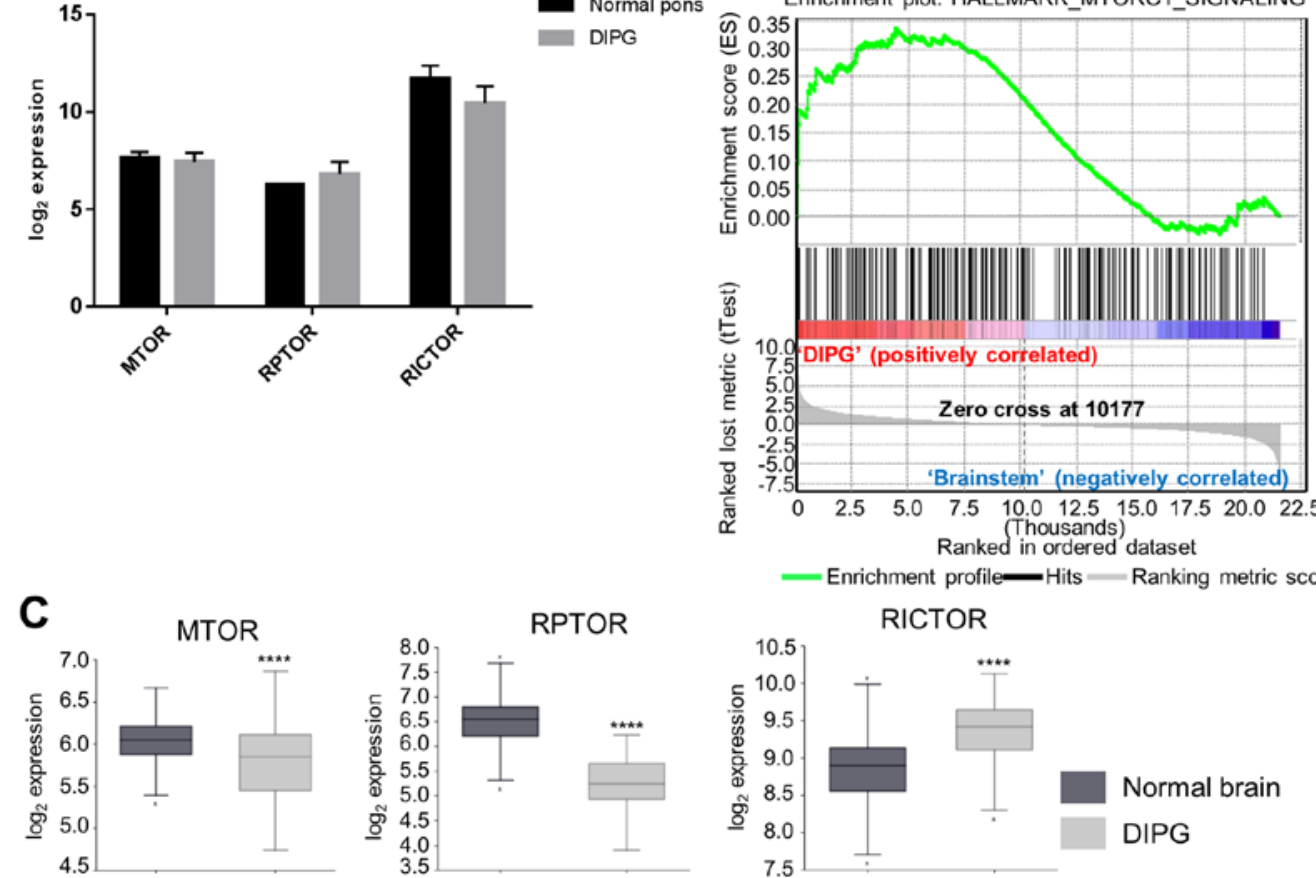

Figure 1. Expression analysis of human DIPG and normal samples. (A) Mean RNA expression levels of MTOR, RPTOR, and RICTOR in normal pons versus DIPG tissue samples from our tumor bank (error bars represent SEM). (B) Gene set enrichment analysis plot for hallmark MTOR signaling in normal versus DIPG tissue samples from our tumor bank. (C) Expression analysis of normal brain versus DIPG samples from the R2 database; plots show mean, interquartile range (shaded area), and total range. ${ }^{* * * * *} \mathrm{p}<0.0001$.
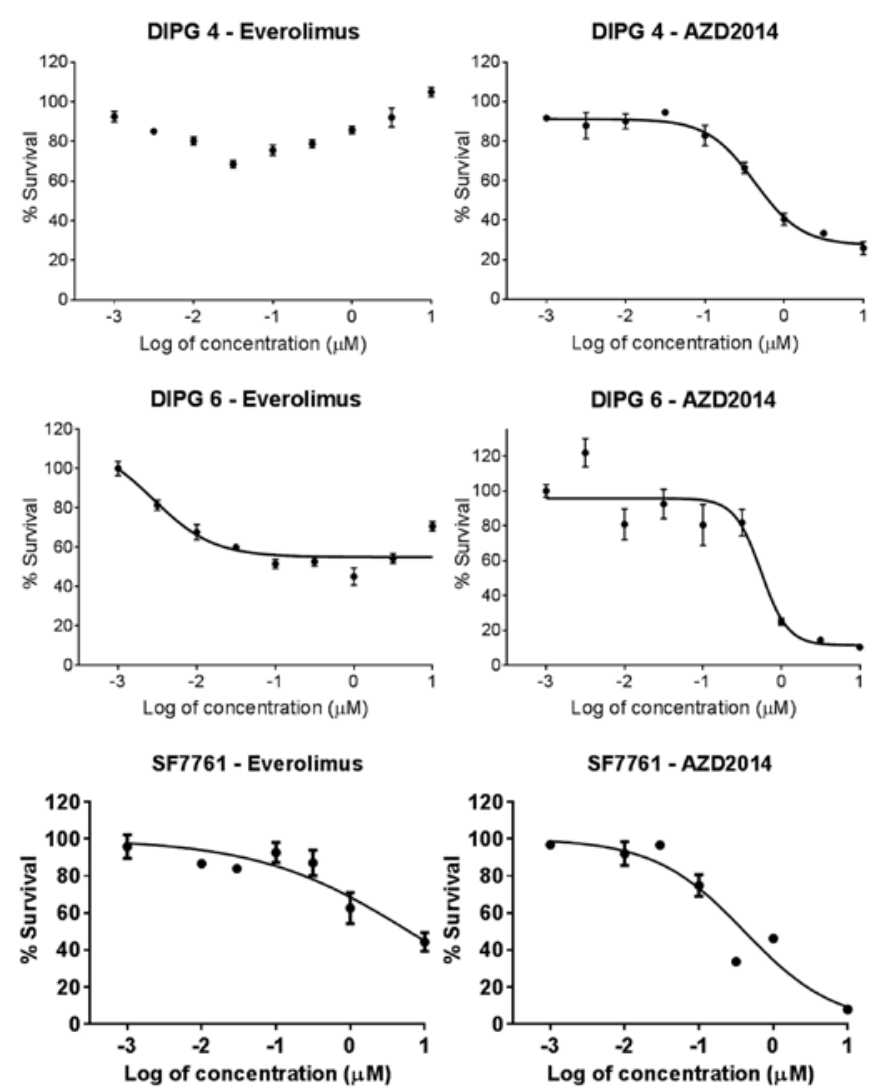

Figure 2. Dose-response curves for three patient-derived DIPG cell lines treated with everolimus versus AZD2014, with cell survival measured by MTS assay (error bars represent SEM); note that a best-fit curve could not be calculated for everolimus in DIPG 4.
Table II. $\mathrm{IC}_{50}$ and $\mathrm{IC}_{90}$ values.

\begin{tabular}{lcclccc}
\hline & \multicolumn{2}{c}{ Everolimus } & & \multicolumn{2}{c}{ AZD2014 } \\
\cline { 2 - 3 } \cline { 5 - 6 } Cell line & $\mathrm{IC}_{50}(\mu \mathrm{M})$ & $\mathrm{IC}_{90}(\mu \mathrm{M})$ & & $\mathrm{IC}_{50}(\mu \mathrm{M})$ & $\mathrm{IC}_{90}(\mu \mathrm{M})$ \\
\hline DIPG 4 & Not reached & Not reached & & 0.425 & 1.90 \\
DIPG 6 & Not reached & Not reached & & 0.552 & 1.30 \\
SF7761 & 6.22 & 1,070 & & 0.410 & 8.86 \\
\hline
\end{tabular}

DIPG samples versus normal pons samples in our tissue bank. Multiple measures showed no significant difference in expression between the two sample sets (Fig. 1A). On hallmark GSEA, however, the MTORC1 signaling pathway was enriched in the DIPG versus normal samples (NES 1.3, $p=0.021$, Fig. 1B). We then compared expression of the same three genes using publically-available sets of normal brain $(\mathrm{n}=172)$ and DIPG $(\mathrm{n}=37)$ samples. MTOR $\left(\mathrm{p}=4.4 \times 10^{-6}\right)$ and RPTOR $\left(\mathrm{p}=4.2 \times 10^{-29}\right)$ were both underexpressed in DIPG compared to normal brain, while RICTOR $\left(p=3.3 \times 10^{-9}\right)$ was overexpressed (Fig. 1C).

MTORC1/2 inhibition decreases cell survival in vitro versus MTORC1 inhibition alone. We measured cell survival after five days of continuous drug exposure by MTS assay for three primary human DIPG cell lines, DIPG 4, DIPG 6, and SF7761 in neurosphere culture. Antitumor efficacy, especially in terms of the $\mathrm{IC}_{90}$, was far greater for AZD2014 compared to everolimus (Fig. 2). For DIPG 4, the $\mathrm{IC}_{50}$ and $\mathrm{IC}_{90}$ were not reached for everolimus and were 0.425 and $1.90 \mu \mathrm{M}$ for AZD2014, 
A

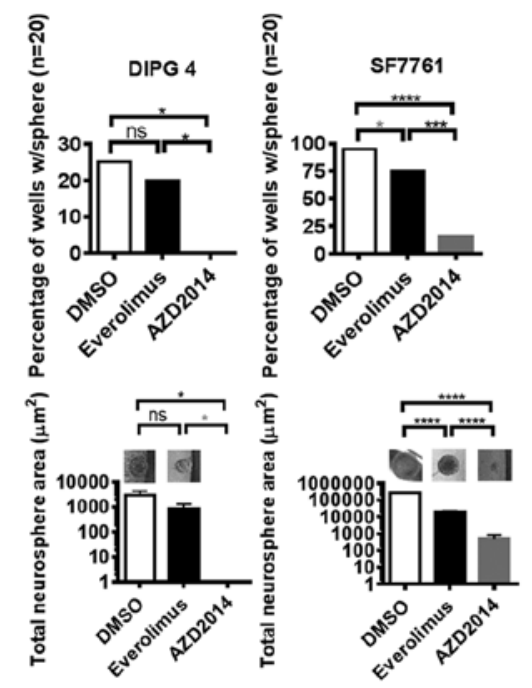

B

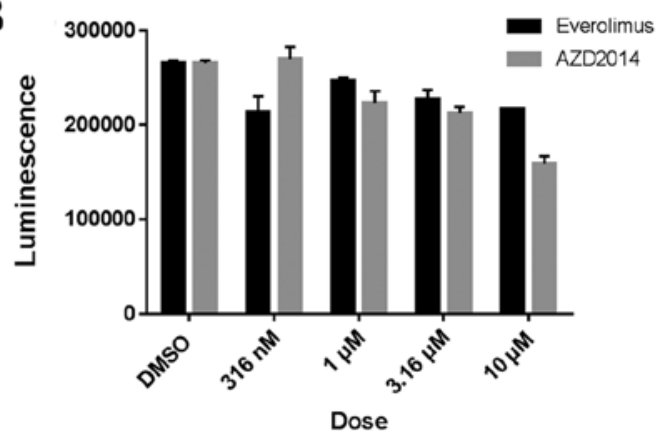

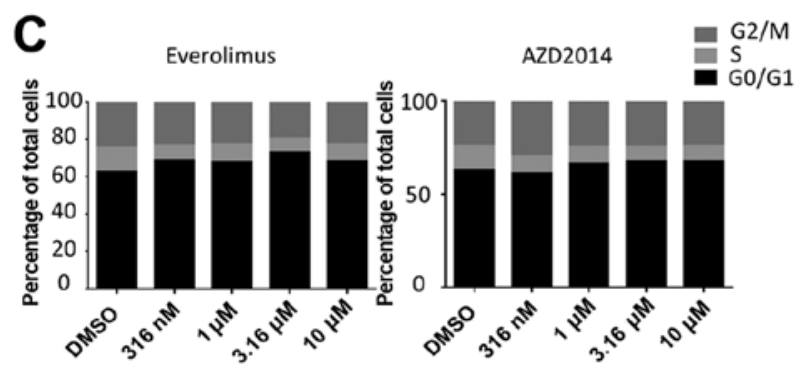
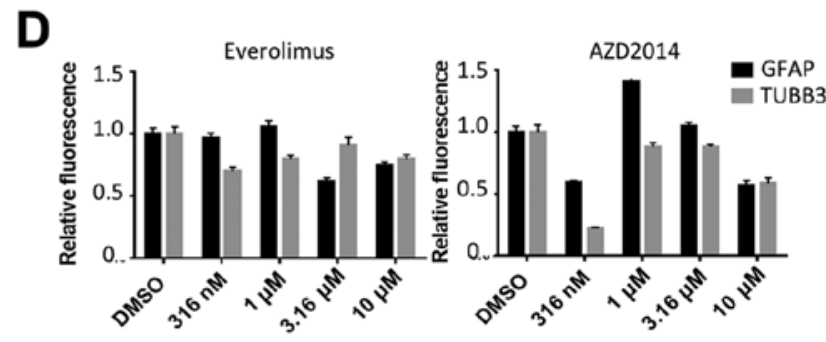

Figure 3. Drug effects on cell self-renewal, apoptosis, cell cycle distribution and differentiation. (A) Top, percentage of wells with an observable neurosphere after exposure to each condition in a neurosphere dilution assay, and bottom, mean neurosphere area after exposure to each condition in a neurosphere dilution assay, with representative neurosphere images (error bars represent SEM). ns, not significant $\mathrm{p}>0.05 ;{ }^{*} \mathrm{p}<0.05 ;{ }^{* * *} \mathrm{p}<0.001 ;{ }^{* * * *} \mathrm{p}<0.0001$. (B) Measurement of apoptosis by caspase 3/7 luminescence assay (error bars represent SEM). (C) Measurement of cell cycle distribution by flow cytometry for propidium iodide. (D) Measurement of GFAP and TUBB3 protein expression by immunofluorescence intensity (error bars represent SEM).

respectively (Table II). For DIPG 6, the $\mathrm{IC}_{50}$ and $\mathrm{IC}_{90}$ were also not reached for everolimus and were 0.552 and $1.30 \mu \mathrm{M}$ for AZD2014. For SF7761, the $\mathrm{IC}_{50}$ and $\mathrm{IC}_{90}$ were 6.22 and $1,070 \mu \mathrm{M}$ for everolimus and 0.410 and $8.86 \mu \mathrm{M}$ for AZD2014.

Cell self-renewal difference but no clear difference in other phenotypic measures. We next examined the general mechanism of action for differences in the effects between the two drugs. When DIPG 4 and SF7761 cells were plated in 96-well format at 10 cells per well and 20 wells per condition to test cell self-renewal capability, those exposed continuously to the $\mathrm{IC}_{75}$ dose of AZD2014 were less likely to form spheres and formed smaller spheres than those exposed to the same concentration of everolimus, or control (Fig. 3A). After $48 \mathrm{~h}$ of treatment, everolimus and AZD2014 both caused a decrease in apoptosis compared to the control, as measured by caspase $3 / 7$ luminescence assay (Fig. 3B); there was no significant difference between the two drugs. We noted no significant difference in cell cycle distribution with increasing doses of either drug as measured by propidium iodide flow cytometry (Fig. 3C). We also saw no clear trend to demonstrate an increase or decrease in glial or neuronal differentiation as measured by GFAP or TUBB3 immunofluorescence, respectively (Fig. 3D).

When we measured induction of senescence in DIPG 4 and SF7761 cells by immunofluorescence for the senescence marker p21, neither compound showed a consistent increase in senescence compared to the control (Fig. 4A). We also measured by staining for the senescence marker $\beta$-galactosidase in DIPG 4 cells; here, both compounds appeared to increase the percentage of senescent cells in a dose-dependent manner, but there was no clear difference between the two (Fig. 4B). AZD2014 and everolimus both induced greater levels of autophagic flux compared to the control, as determined by western blotting for LC3-II, an autophagy biomarker (Fig. 4C). Neither compound induced consistently more autophagy than the other, however.

Inhibition of AKT phosphorylation is necessary and sufficient for the effect of AZD2014 on DIPG. We next set out to determine the specific mechanism of action differentiating AZD2014 from everolimus, focusing on AKT, which is activated when phosphorylated by MTORC2 but is upstream of MTORC1. We found that increasing doses of AZD2014 caused a dose-dependent decrease in pAKT relative to total AKT, as determined by western blotting (Fig. 5A). We saw no such decrease with everolimus. We then used a direct AKT inhibitor, MK-2206, and demonstrated that exposing DIPG 4 and SF7761 cells in neurosphere culture to this drug for five days resulted in $\mathrm{IC}_{50}$ levels of $2.60 \mu \mathrm{M}$ in DIPG 4 and $1.08 \mu \mathrm{M}$ in SF7761 cells (Fig. 5B). These values were higher but within the range of those obtained with AZD2014. We then transfected DIPG 4 cells with a cDNA for a mutant form of AKT (S473D) intending to confer constitutive activation. Compared to the wild-type, the transfected cells exhibited 

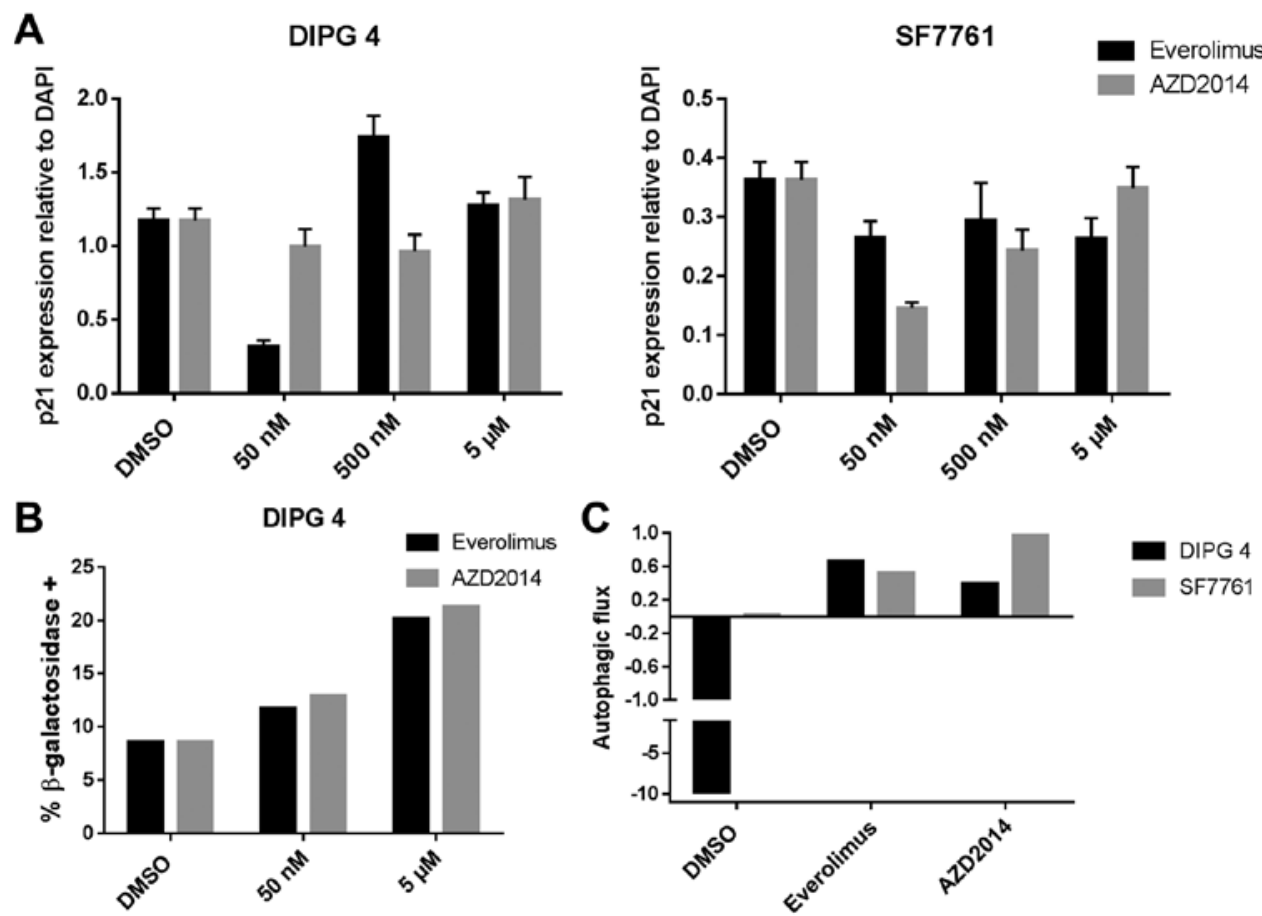

Figure 4. Drug effects on senescence and autophagy. (A) Effect of everolimus and AZD2014 on senescence, as measured by the ratio of immunofluorescence intensity of p21 compared to DAPI (error bars represent SEM). (B) Effects of everolimus and AZD2014 on senescence, as measured by the percentage of cells staining positive for $\beta$-galactosidase. (C) Effects of everolimus and AZD2014 on autophagy, as measured by autophagic flux, defined as the increase in LC3-II:tubulin western blot intensity ratio with the stated condition as compared to the stated condition plus chloroquine (an autophagy inhibitor).
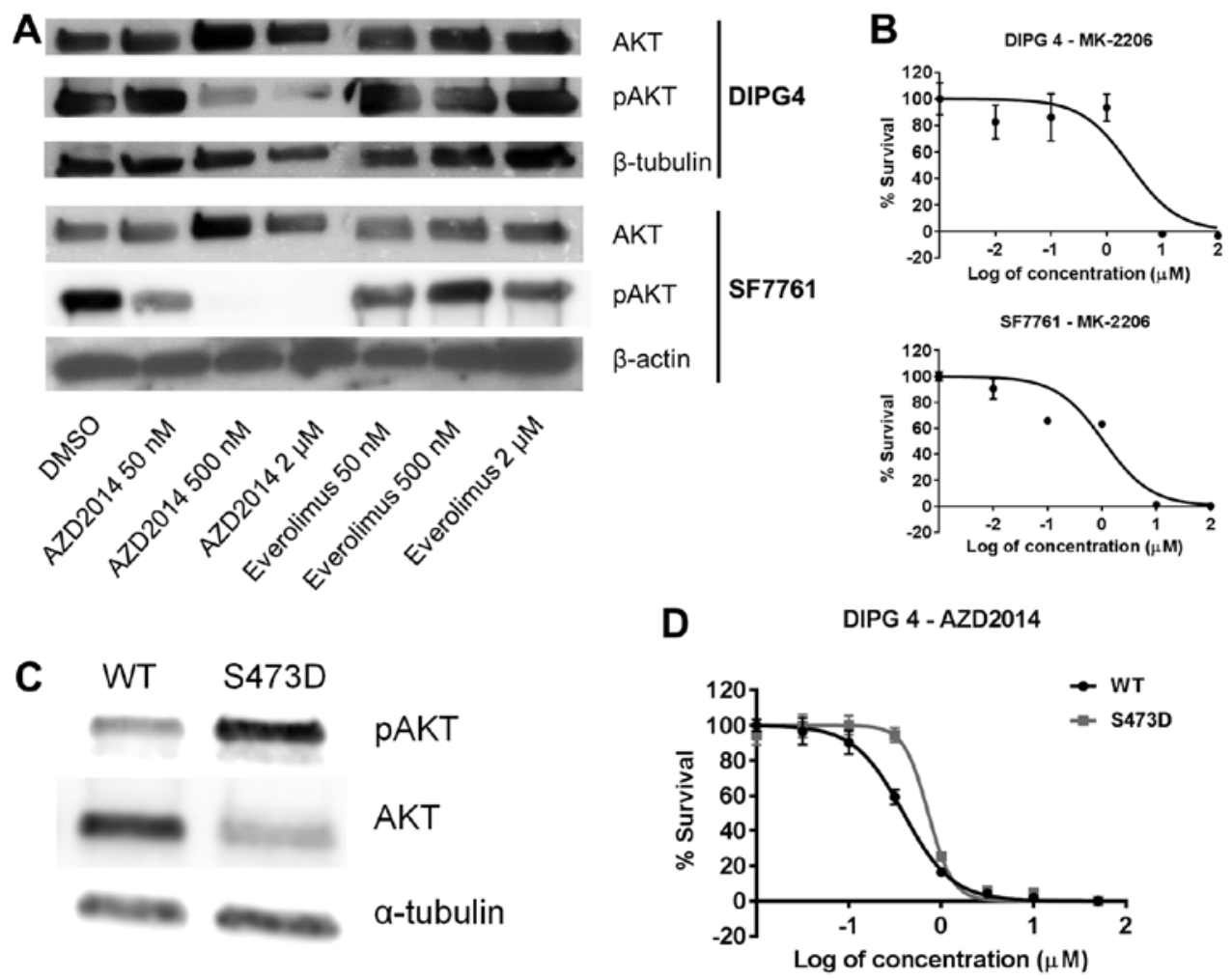

Figure 5. Molecular analyses of drug effect. (A) Western blot showing total AKT and pAKT expression. (B) Dose-response curves for DIPG 4 and SF7761 cells treated with the small molecule AKT inhibitor MK-2206 (error bars represent SEM). (C) Western blot showing the increase in pAKT after transfection with cDNA for S473D-mutant AKT, conferring constitutive activity. (D) Dose-response curves for wild-type (WT) and S473D-mutant AKT-transfected DIPG 4 cells treated with AZD2014 (error bars represent SEM).

increased apparent pAKT (due to the S473D mutation, which binds the phospho-AKT antibody), accompanied by a decrease in unphosphorylated AKT, suggesting that total AKT (measured by the sum of the pAKT and AKT bands) remained 
A

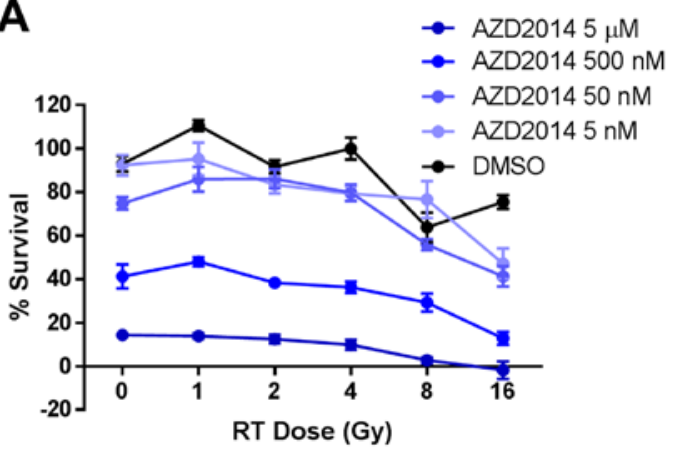

B

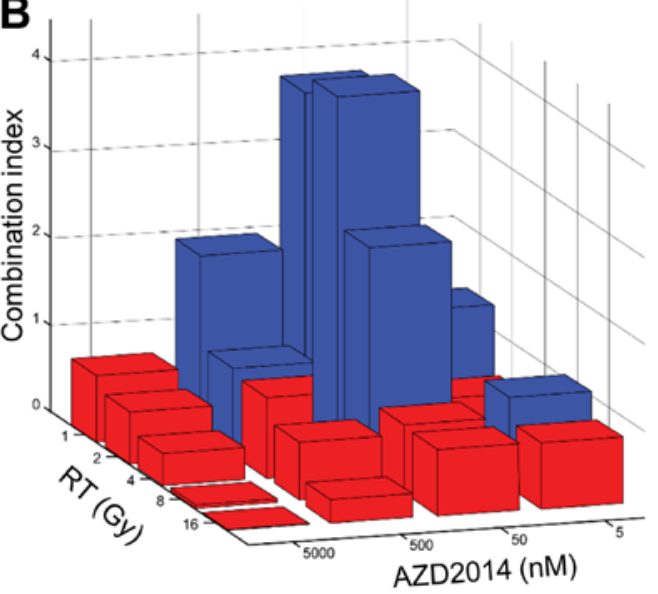

Dose combination: $\square$ Synergistic $\square$ Antagonistic

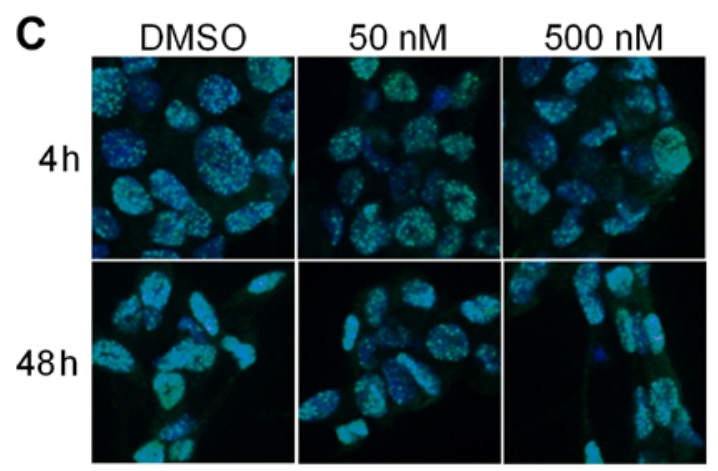

Figure 6. Analysis of AZD2014-RT combination. (A) Dose-response curves for SF7761 cells treated with cesium radiation and AZD2014 (error bars represent SEM). (B) Combination index analysis showing the therapeutic relationship for each dose combination $(<1$, synergistic; $>1$, antagonistic). (C) Immunofluorescence staining for $\gamma \mathrm{H} 2 \mathrm{AX}$ of SF7761 cells treated with RT (4 Gy) following 4-or 48-h treatment with vehicle or AZD2014 at $50 \mathrm{nM}$ or $500 \mathrm{nM}$ dose levels. RT, radiation therapy.

relatively constant between the wild-type and S473D cells in the experiment. Transfection with S473D thus accurately models the phosphorylation of AKT by MTORC2 (Fig. 5C). Cells transfected with S473D mutant AKT demonstrated resistance to AZD2014 as compared to wild-type cells, with an increase in $\mathrm{IC}_{50}$ from 0.390 to $0.724 \mu \mathrm{M}$ (p<0.01, Fig. 5D), strongly suggesting that AZD2014 operates at least in part by inhibiting AKT activation through MTORC2 phosphorylation.

AZD2014 has a variable therapeutic relationship with $R T$ in $D I P G$ depending on dosing. We then tested the therapeutic relationship between AZD2014 and RT, since RT is the current standard of care treatment in DIPG. Because DIPG 4 and DIPG 6 are derived from previously irradiated tumor samples and resistant to RT, we treated radiation-naïve SF7761 cells with increasing doses of AZD2014 and exposed them to various doses of cesium RT in one fraction. We demonstrated a dose-dependent decrease in cell survival with both treatments as measured by MTS assay (Fig. 6A). Maximal cell killing with RT alone was only in the range of $40 \%$, even though SF7761 is our most radiosensitive cell line, because the single fraction delivered is well below the total dose delivered clinically over many fractions. We then determined the therapeutic relationship at each dose combination by the Chou Talalay method (19) to calculate combination indices. The relationship was variable but was generally synergistic (combination index $<1)$ at higher doses of each treatment and antagonistic (combination index $>1$ ) at lower doses (Fig. 6B). We found that, while AZD2014 and RT were synergistic in killing cells at high doses, the level of dsDNA damage caused by this combination did not exceed the damage caused by RT alone as assessed by $\gamma \mathrm{H} 2 \mathrm{AX}$ staining (Fig. 6C).

AZD2014 has variable therapeutic relationships with FDA-approved chemotherapy agents, including synergy with ponatinib. Finally, we conducted a drug screen of all FDA-approved chemotherapy agents in combination with AZD2014, using SF7761 in a 96-well format (data not shown; goo.gl/3XCCqk). Aside from antagonistic relationships with microtubule inhibitors, there was no clear trend to AZD2014's therapeutic relationship with any category of drug. AZD2014 did show synergistic relationships with drugs from multiple classes, however, including microtubule stabilizers, topoisomerase inhibitors, and tyrosine kinase inhibitors. We then validated our screening finding of a potentially synergistic relationship between AZD2014 and the multikinase inhibitor ponatinib using multiple dose levels of the AZD2014-ponatinib combination in DIPG 4, DIPG 6, and SF7761 (Fig. 7A). We demonstrated synergy between AZD2014 and ponatinib at most dose levels in all three cell lines, especially dose levels exceeding the combined $\mathrm{IC}_{50}$ of the two agents (Fig. 7B).

\section{Discussion}

In this study, we demonstrated that MTORC1/2 inhibition shows greatly increased antitumor efficacy in a panel of patient-derived DIPG cell lines compared to MTORC1 inhibition alone, which showed little to no effect. The phenotype behind this advantage appears to be a decrease in cell self-renewal; assays testing other potential general mechanisms of action showed no significant or consistent differences between the two drugs. On a molecular level, the decrease in AKT phosphorylation caused by MTORC2 inhibition appears to be sufficient and at least partially necessary to the augmented tumor inhibition when MTORC2 is targeted. This phenotype and molecular mechanism are consistent with the known role of AKT in cancer cell self-renewal (20-22). Finally, we showed that AZD2014 has the potential to act synergistically with RT, as well as with cytotoxic and targeted chemotherapeutic agents of various classes.

It is unclear whether the efficacy of AZD2014 in this study depends on overexpression of MTORC2 components. Expression levels of MTOR, RPTOR, and RICTOR were similar in the DIPG samples in our tumor bank compared 


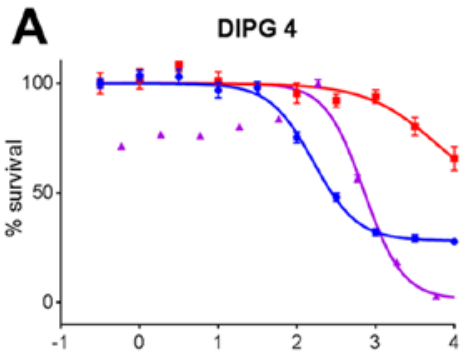

Log of concentration (nM)

B

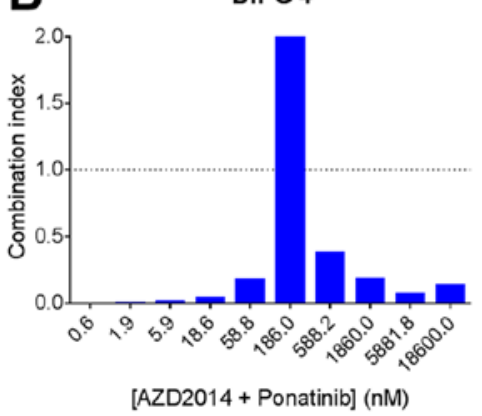

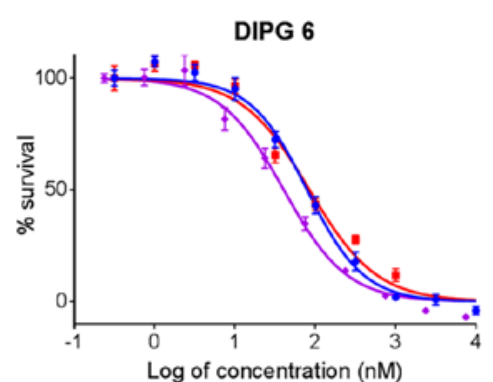

DIPG 6

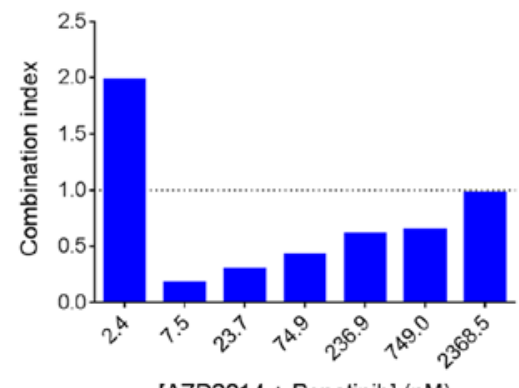

[AZD2014 + Ponatinib] (nM)

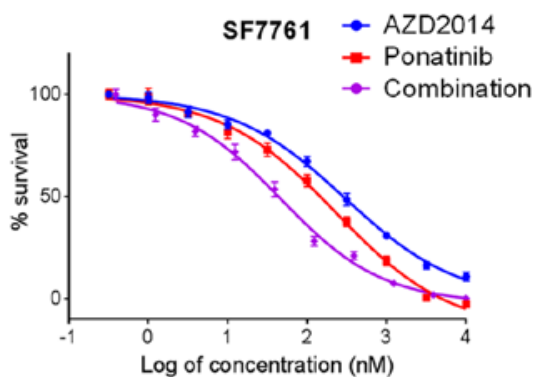

SF7761

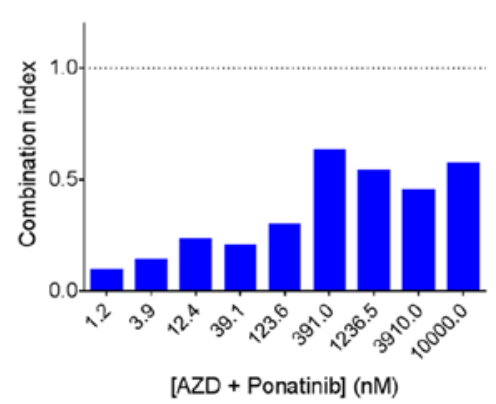

Figure 7. Analysis of AZD2014-chemotherapy combination. (A) Dose-response curves for DIPG cells treated with AZD2014, ponatinib, and the combination (error bars represent SEM). (B) Combination index analysis showing the therapeutic relationship for each dose combination $(<1$, synergistic; $>1$, antagonistic).

to levels in normal pons, although the MTOR pathway was enriched in DIPG on GSEA. MTOR and RPTOR were underexpressed in a large public dataset of DIPG samples, while RICTOR was overexpressed, which could contribute to an explanation for the difference seen between compounds, since RICTOR is unique to MTORC2. It should be noted in this analysis, however, that the available comparison group was tissue samples from throughout the brain, not the pons alone. In terms of previous studies, a large study of 43 DIPG samples that examined copy number abnormalities and expression profiles did not identify MTOR in any of the abnormalities (23). This study did find AKT as a potential gene of interest in focal recurrent gains, however. Another group working with a genetically engineered mouse model of DIPG found that AKT was overexpressed in cell lines derived from this model, and when they conducted a high-throughput drug screen against these cells, they identified antitumor activity in a multikinase inhibitor that decreases levels of pAKT (24). These findings support our results showing that AKT inhibition through MTORC1 and MTORC2 is necessary and sufficient to the antitumor effect of AZD2014, and that this is the reason for everolimus' lack of activity.

RT is currently the only effective primary therapy in DIPG, and clinical trial data are also emerging to suggest its utility at recurrence as well $(25,26)$. Therefore, the therapeutic relationship with $\mathrm{RT}$ will be relevant to any new targeted drug proposed. Our findings suggest that AZD2014 and RT have a synergistic therapeutic relationship at higher doses of each. These higher RT doses are used in DIPG, and AZD2014 levels at and above the $\mathrm{IC}_{50}$ will also be necessary to achieve therapeutic effect. We did not observe greater levels of dsDNA damage from the combination versus RT alone and continue to investigate the underlying mechanism of synergy. The prior study examining this combination in adult glioblastoma found that the reason for synergy is most likely inhibition of
DNA repair (13). On our chemotherapy combination screen, AZD2014 also appeared to show synergy with several other DNA damaging agents. Another candidate drug from our chemotherapy screen, ponatinib, primarily targets BCR-ABL but also inhibits VEGFR, PDGFR, and EGFR (27), all of which have relevance to DIPG (28). Ponatinib may also inhibit AKT (29), raising another possible mechanism of synergy with AZD2014. Given that development of resistance to targeted therapies is a major issue with their efficacy, using combinations such as this to target multiple key oncogenic pathways may be crucial to successful DIPG treatment. Our consistent results with ponatinib in each of the lines validates our screening method. The availability of these preclinical models in DIPG is of great translational value. Besides their lack of efficacy, the drugs used in previous DIPG clinical trials have also caused harm through adverse effects, mandating that future treatments undergo rational preclinical testing before they are deemed worthy of the risk they carry for patients.

Our study has several limitations. We did not show that AZD2014 reverts to an everolimus-like dose-response curve with constitutive AKT activation, although the difference in $\mathrm{IC}_{50}$ was statistically significant. This may be because AZD2014 was still able to achieve some level of AKT inhibition due to imperfect transfection efficiency in this experiment. Also, AZD2014's blood-brain barrier (BBB) penetration is unknown, but given the concerns with drug penetration in DIPG in general, it is likely that local delivery methods that bypass the BBB, such as convection-enhanced delivery, will at least partially obviate this consideration. Finally, we do not yet have in vivo validation of our findings. However, while our study was under review, Miyahara et al published their findings on the effect of another MTORC1/2 inhibitor, TAK228, in DIPG, including its efficacy in a patient-derived xenograft model (30). Our study delves further into the molecular mechanism of action and potential combination chemotherapy 
approaches, and together, these two studies on different MTORC1/2 inhibitors provide strong preclinical rationale for this strategy in DIPG.

In conclusion, the present study demonstrates that DIPG does not respond preclinically to MTORC1 inhibition alone but does respond well to combined MTORC1/2 inhibition, due to the inhibitory effect of MTORC2 on AKT. AZD2014, an MTORC1/2 inhibitor, shows synergy with RT and with selected chemotherapy agents in DIPG. This strategy should be studied further as a potential component of combinatorial approaches to treatment of this currently incurable tumor.

\section{Acknowledgements}

The authors wish to thank Radu Moldovan and Alireza Hemmati of the Anschutz Medical Campus Advanced Light Microscopy Core Facility for their support. This study was supported by a grant from the Morgan Adams Foundation. A.L.G. is the Luke's Army Pediatric Cancer Research Fund St. Baldrick's Fellow and a Hyundai Hope on Wheels Young Investigator.

\section{References}

1. Fangusaro J: Pediatric high-grade gliomas and diffuse intrinsic pontine gliomas. J Child Neurol 24: 1409-1417, 2009.

2. Massimino M, Spreafico F, Biassoni V, Simonetti F, Riva D, Trecate G, Giombini S, Poggi G, Pecori E, Pignoli E, et al: Diffuse pontine gliomas in children: Changing strategies, changing results? A mono-institutional 20 -year experience. J Neurooncol 87: 355-361, 2008.

3. Green AL and Kieran MW: Pediatric brainstem gliomas: New understanding leads to potential new treatments for two very different tumors. Curr Oncol Rep 17: 436, 2015.

4. Hashizume R, Ozawa T, Gryaznov SM, Bollen AW, Lamborn KR, Frey WH II and Deen DF: New therapeutic approach for brain tumors: Intranasal delivery of telomerase inhibitor GRN163. Neuro-oncol 10: 112-120, 2008.

5. Goodwin CR, Xu R, Iyer R, Sankey EW, Liu A, Abu-Bonsrah N, Sarabia-Estrada R, Frazier JL, Sciubba DM and Jallo GI: Local delivery methods of therapeutic agents in the treatment of diffuse intrinsic brainstem gliomas. Clin Neurol Neurosurg 142 120-127, 2016.

6. Barua NU, Lowis SP, Woolley M, O'Sullivan S, Harrison R and Gill SS: Robot-guided convection-enhanced delivery of carboplatin for advanced brainstem glioma. Acta Neurochir (Wien) 155: 1459-1465, 2013.

7. Cohen K, Jones A, Raabe E and Pearl M: Highly selective intra-arterial chemotherapy for the treatment of progressive diffuse intrinsic pontine gliomas (DIPG). In: 20th International Conference on Brain Tumor Research and Therapy NeuroOncology, Lake Tahoe, CA. Neurooncology 16: iii29, 2014.

8. Zeng Z, Sarbassov D, Samudio IJ, Yee KW, Munsell MF, Ellen Jackson C, Giles FJ, Sabatini DM, Andreeff M and Konopleva M: Rapamycin derivatives reduce mTORC2 signaling and inhibit AKT activation in AML. Blood 109: 3509-3512, 2007.

9. Guertin DA, Stevens DM, Thoreen CC, Burds AA, Kalaany NY, Moffat J, Brown M, Fitzgerald KJ and Sabatini DM: Ablation in mice of the mTORC components raptor, rictor, or mlST8 reveals that mTORC2 is required for signaling to Akt-FOXO and PKCalpha, but not S6K1. Dev Cell 11: 859-871, 2006.

10. Guertin DA and Sabatini DM: Defining the role of mTOR in cancer. Cancer Cell 12: 9-22, 2007.

11. Akhavan D, Cloughesy TF and Mischel PS: mTOR signaling in glioblastoma: Lessons learned from bench to bedside. Neuro-oncol 12: 882-889, 2010.

12. Galanis E, Buckner JC, Maurer MJ, Kreisberg JI, Ballman K, Boni J, Peralba JM, Jenkins RB, Dakhil SR, Morton RF, et al; North Central Cancer Treatment Group: Phase II trial of temsirolimus (CCI-779) in recurrent glioblastoma multiforme: A North Central Cancer Treatment Group Study. J Clin Oncol 23: 5294-5304, 2005.
13. Kahn J, Hayman TJ, Jamal M, Rath BH, Kramp T, Camphausen K and Tofilon PJ: The mTORC1/mTORC2 inhibitor AZD2014 enhances the radiosensitivity of glioblastoma stem-like cells. Neuro-oncol 16: 29-37, 2014

14. Schwartzentruber J, Korshunov A, Liu XY, Jones DT, Pfaff E, Jacob K, Sturm D, Fontebasso AM, Quang DA, Tönjes M, et al: Driver mutations in histone H3.3 and chromatin remodelling genes in paediatric glioblastoma. Nature 482: 226-231, 2012.

15. Buczkowicz P, Hoeman C, Rakopoulos P,Pajovic S, Letourneau L, Dzamba M, Morrison A, Lewis P, Bouffet E, Bartels U, et al: Genomic analysis of diffuse intrinsic pontine gliomas identifies three molecular subgroups and recurrent activating ACVR1 mutations. Nat Genet 46: 451-456, 2014.

16. Mehta S, Huillard E, Kesari S, Maire CL, Golebiowski D, Harrington EP, Alberta JA, Kane MF, Theisen M, Ligon KL, et al: The central nervous system-restricted transcription factor Olig2 opposes p53 responses to genotoxic damage in neural progenitors and malignant glioma. Cancer Cell 19: 359-371, 2011.

17. Nagaraja S, Vitanza NA, Woo PJ, Taylor KR, Liu F, Zhang L, Li M, Meng W, Ponnuswami A, Sun W, et al: Transcriptional dependencies in diffuse intrinsic pontine glioma. Cancer Cell 31: 635-652 e636, 2017.

18. Rodrik-Outmezguine VS, Chandarlapaty S, Pagano NC, Poulikakos PI, Scaltriti M, Moskatel E, Baselga J, Guichard S and Rosen N: mTOR kinase inhibition causes feedback-dependent biphasic regulation of AKT signaling. Cancer Discov 1: 248-259, 2011.

19. Chou TC: Drug combination studies and their synergy quantification using the Chou-Talalay method. Cancer Res 70: 440-446, 2010.

20. Bahena-Ocampo I, Espinosa M, Ceballos-Cancino G, Lizarraga F, Campos-Arroyo D, Schwarz A, Garcia-Lopez P, Maldonado V and Melendez-Zajgla J: miR-10b expression in breast cancer stem cells supports self-renewal through negative PTEN regulation and sustained AKT activation. EMBO Rep 17: 1081, 2016.

21. Lin Y, Yang Y, Li W, Chen Q, Li J, Pan X, Zhou L, Liu C, Chen C, $\mathrm{He} \mathrm{J}$, et al: Reciprocal regulation of Akt and Oct4 promotes the self-renewal and survival of embryonal carcinoma cells. Mol Cell 48: 627-640, 2012.

22. Singh S, Trevino J, Bora-Singhal N, Coppola D, Haura E, Altiok $S$ and Chellappan SP: EGFR/Src/Akt signaling modulates Sox 2 expression and self-renewal of stem-like side-population cells in non-small cell lung cancer. Mol Cancer 11: 73, 2012.

23. Paugh BS, Broniscer A, Qu C, Miller CP, Zhang J, Tatevossian RG, Olson JM, Geyer JR, Chi SN, da Silva NS, et al: Genome-wide analyses identify recurrent amplifications of receptor tyrosine kinases and cell-cycle regulatory genes in diffuse intrinsic pontine glioma. J Clin Oncol 29: 3999-4006, 2011.

24. Halvorson KG, Barton KL, Schroeder K, Misuraca KL, Hoeman C, Chung A, Crabtree DM, Cordero FJ, Singh R, Spasojevic I, et al: A high-throughput in vitro drug screen in a genetically engineered mouse model of diffuse intrinsic pontine glioma identifies BMS-754807 as a promising therapeutic agent. PLoS One 10: e0118926, 2015.

25. Fontanilla HP, Pinnix CC, Ketonen LM, Woo SY, Vats TS Rytting ME, Wolff JE and Mahajan A: Palliative reirradiation for progressive diffuse intrinsic pontine glioma. Am J Clin Oncol 35: 51-57, 2012.

26. Wolff JE, Rytting ME, Vats TS, Zage PE, Ater JL, Woo S, Kuttesch J, Ketonen L and Mahajan A: Treatment of recurrent diffuse intrinsic pontine glioma: The MD Anderson Cancer Center experience. J Neurooncol 106: 391-397, 2012.

27. Frankfurt O and Licht JD: Ponatinib - a step forward in overcoming resistance in chronic myeloid leukemia. Clin Cancer Res 19: 5828-5834, 2013.

28. Jansen MH, van Vuurden DG, Vandertop WP and Kaspers GJ: Diffuse intrinsic pontine gliomas: A systematic update on clinical trials and biology. Cancer Treat Rev 38: 27-35, 2012.

29. Kim DH, Kwak Y, Kim ND and Sim T: Antitumor effects and molecular mechanisms of ponatinib on endometrial cancer cells harboring activating FGFR2 mutations. Cancer Biol Ther 17: 65-78, 2016.

30. Miyahara H, Yadavilli S, Natsumeda M, Rubens JA, Rodgers L, Kambhampati M, Taylor IC, Kaur H, Asnaghi L, Eberhart CG, et al: The dual mTOR kinase inhibitor TAK228 inhibits tumorigenicity and enhances radiosensitization in diffuse intrinsic pontine glioma. Cancer Lett 400: 110-116, 2017.

This work is licensed under a Creative Commons Attribution-NonCommercial-NoDerivatives 4.0 International (CC BY-NC-ND 4.0) License. 